\title{
Correction to: The first authenticated record of the pangolin tick Amblyomma javanense (Acari: Ixodidae) in Singapore, with notes on its biology and conservation
}

\author{
Mackenzie L. Kwak ${ }^{1}$. Chia-Da Hsu² - Guillaume Douay² • Ali Anwar Ahmad²
}

Published online: 18 October 2018

๑) Springer Nature Switzerland AG 2018

\section{Correction to: Experimental and Applied Acarology https://doi.org/10.1007/s10493-018-0310-7}

Due to an unfortunate turn of events, the surname of the third author appeared incorrectly in the original publication as it should have read Douay. The original article has been corrected, and the proper representation of the authors' names and their affiliation is also listed here.

The original article can be found online at https://doi.org/10.1007/s10493-018-0310-7.

Mackenzie L. Kwak

mackenziekwak@gmail.com

1 Department of Biological Science, National University of Singapore, 16 Science Drive 4,

Singapore 117558, Republic of Singapore

2 Department of Conservation, Research and Veterinary Services, Wildlife Reserves Singapore, 80 Mandai Lake Road, Singapore 729826, Republic of Singapore 